\title{
Development of Clinician-Reported Outcome (ClinRO) and Patient-Reported Outcome (PRO) Measures for Eyebrow, Eyelash and Nail Assessment in Alopecia Areata
}

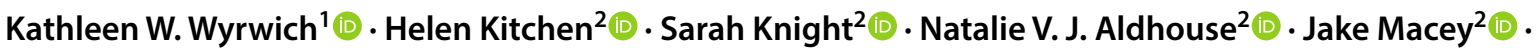

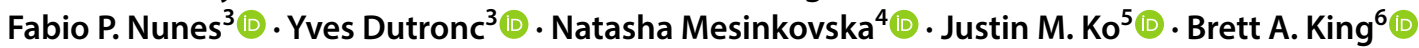

Published online: 17 August 2020

(c) The Author(s) 2020

\begin{abstract} transcripts were qualitatively analyzed.

\section{Introduction}

Understanding and evaluating the various manifestations of diseases and patients' treatment experiences is critical for the development and evaluation of new therapies [1]. Current clinical assessment guidelines for alopecia areata (AA) focus on scalp hair loss, with secondary assessment of "body hair loss" and "nail involvement" [2-4]. However, it is known that AA can also cause eyebrow and eyelash hair loss $[5,6]$ and that the loss of these defining facial features is physically and psychologically bothersome to patients $[7,8]$.
\end{abstract}

Background Eyebrow and eyelash hair loss and nail damage—in addition to scalp hair loss—are important signs/symptoms of alopecia areata (AA) to patients and deserve assessment in AA clinical trials.

Objectives Our objective was to develop clinician-reported outcome (ClinRO) and patient-reported outcome (PRO) measures and accompanying photoguides to aid in the assessment of AA-related eyebrow, eyelash and nail signs/symptoms.

Methods Iterative rounds of qualitative, semi-structured interviews were conducted with US expert dermatologists and North American patients with AA. Patients with eyebrow, eyelash and nail involvement were purposefully sampled. Interview

Results Dermatologists $(n=10)$ described eyebrow and eyelash loss as concerning for affected patients and, along with nail appearance, as deserving assessment. Dermatologist data informed the development of single item, 4-point Likert-type ClinRO and PRO measures of current eyebrow loss, eyelash loss and nail appearance and a PRO measure of eye irritation. Patients ( $n=45$, age 15-72 years) confirmed the importance and relevance of these signs/symptoms. Interim revision resulted in measures that were understood by and relevant to patients. Dermatologists $(n=5)$ and patients $(n=10$, age 21-54 years) participated in the development of the eyebrow, eyelash and nail photoguides and confirmed that they included photos that appropriately represented different severity levels and were helpful to derive and standardize ratings across raters.

Conclusions The ClinRO and PRO measures for eyebrow, eyelash and nail appearance, with their accompanying photoguides and the PRO Measure for Eye Irritation provide clear and meaningful assessments of outcomes important to patients with AA.

Electronic supplementary material The online version of this article (https://doi.org/10.1007/s40257-020-00545-9) contains supplementary material, which is available to authorized users.

Brett A. King

brett.king@yale.edu

Extended author information available on the last page of the article
Eyebrows are also important for facial recognition [9]. Additionally, nail changes are not uncommon, affecting 7-66\% (average $30 \%$ ) of patients with AA [10].

Fit-for-purpose clinical outcome assessments (COAs) are required to evaluate the efficacy of treatment on these key non-scalp signs/symptoms in AA clinical trials and to complement clinician and patient assessments of scalp hair loss $[11,12]$. However, to our knowledge, no publicly available outcome measures to evaluate eyebrow hair loss, eyelash hair loss and nail damage have been developed in conjunction with industry guidelines [13].

This series of cross-sectional, non-interventional, qualitative interview studies aimed to develop clinically relevant and usable: (1) clinician-reported outcome (ClinRO) measures, (2) patient-reported outcome (PRO) measures and (3) accompanying photoguides to guide clinicians and patients to derive scores related to these measures and help standardize ratings by providing clear visual instruction for assessment of these non-scalp signs of AA. 


\section{Key Points}

Iterative rounds of qualitative interviews with dermatologists and patients in North America confirmed that eyebrow hair loss, eyelash hair loss, nail damage and eye irritation are bothersome signs/symptoms of alopecia areata (AA) that warrant assessment in clinical trials.

The content validity of newly developed clinicianreported outcome (ClinRO) and patient-reported outcome (PRO) measures of these signs/symptoms, along with corresponding photoguides, was confirmed during these interviews; quantitative studies to confirm their psychometric properties are ongoing.

The ClinRO and PRO measures may be used in similar contexts and populations to provide clinically meaningful assessments that reflect patients' and dermatologists' perspectives of AA.

\section{Methods}

\subsection{Qualitative Approach and Research Perspective}

This study was designed to develop novel PRO and ClinRO measures through methodology aligned with the US FDA PRO Guidance for Industry [13] and good research practice recommendations (Fig. 1) [14]. Draft measures were initially developed by expert clinicians (FPN, BAK) with input from COA experts (KWW, HK, SK, NVJA). Iterative sets of qualitative interviews and a small panel meeting (SPM) comprising expert dermatologists were then conducted to develop the measures and accompanying photoguides.

\subsection{Interview Conduct}

The study protocol was approved by Western Institutional Review Board (ref. \#20171820). All interviews were conducted between October 2017 and September 2018 by a qualitative interviewer trained in COA development techniques. Interviews were audio-recorded and transcribed verbatim. Separate semi-structured interview guides were used in each interview set/round and included concept elicitation (open-ended and probed questioning to systematically explore dermatologists' and patients' insights into AA) and/ or cognitive debriefing $[14,15]$ to evaluate acceptance and interpretation of the ClinRO and PRO measures.

\subsubsection{Sampling}

Expert dermatologists practicing in the USA were identified by their involvement in contemporary research and treatment of patients with AA. Eligible patients in North America were identified by dermatologists at the University of California Irvine, Yale University, Northwest Dermatology Research Center, SKiN Centre for Dermatology and Dawes Fretzin Dermatology. Patients were eligible for inclusion if they had severe AA, determined by present or recent experience of $\geq 50 \%$ scalp hair loss. Purposive sampling targeted the inclusion of patients with current or previous eyebrow, eyelash and/or nail involvement to ensure that the PRO measures and accompanying photoguides were reviewed by patients with relevant AA experience. Full eligibility criteria for each round of the study are provided in the Online Resource 1.

\subsection{Analysis}

Interview transcripts were coded using ATLAS.ti version 7.5. Thematic analysis [16] took a phenomenological approach, focusing on the perceptions, feelings and lived experiences of participants [17]. Conceptual saturation, the point at which no new concept-relevant information emerges [18], was explored to guide patient interview sampling and analysis. Saturation was achieved in round 1, indicating understanding of the elicited sign/symptom concepts [14, 19]; round 2 interviews then sought to refine and confirm the PRO and ClinRO measures. Cognitive debriefing data were subject to framework analysis [20] to identify the relevance and appropriateness of item wording, response options and recall period.

\section{Results}

\subsection{Development of Clinician-Reported Outcome (ClinRO) and Patient-Reported Outcome (PRO) Measures}

\subsubsection{Sample}

Ten expert dermatologists, with 6-38 years (mean 21.2) of experience in managing patients with AA, and 45 patients with AA, aged 15-72 years (mean 33.5), participated in these interviews (round $1[n=30]$; round $2[n=15]$ ). A diverse patient sample was recruited: $58 \%(n=26)$ of participants were female, $44 \%(n=20)$ were non-Caucasian, $53 \%$ $(n=24)$ reported a high school diploma or lower education level and 78\% ( $n=35)$ had partial/full eyebrow and/or eyelash hair loss. Full clinical and demographic characteristics are provided in Online Resource 2. 


\section{Iterative development of patient-reported outcome (PRO) and clinician-reported outcome (ClinRO) measures}

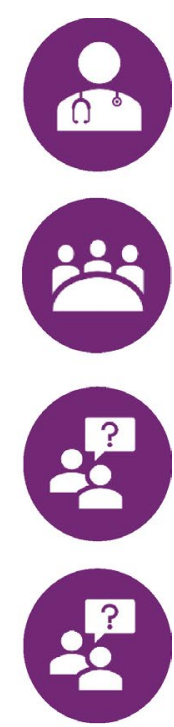

1. Dermatologist interviews: 60-minute, one-on-one telephone interviews with 10 expert dermatologists to iteratively develop draft ClinRO and PRO measures

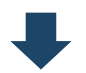

2. Small panel meeting (SPM): Two of the experts previously interviewed resolved discrepancies in findings and finalized draft ClinRO and PRO measures for review with patients

3. Patient interviews (Round 1): 90-minute, one-on-one, face-to-face interviews with 30 patients to understand AA signs/symptoms and review ClinRO and PRO measures

4. Patient interviews (Round 2): 90-minute, one-to-one, face-to-face interviews with 15 patients with AA to review revisions to PRO measures following Round 1

Final PRO and ClinRO measures
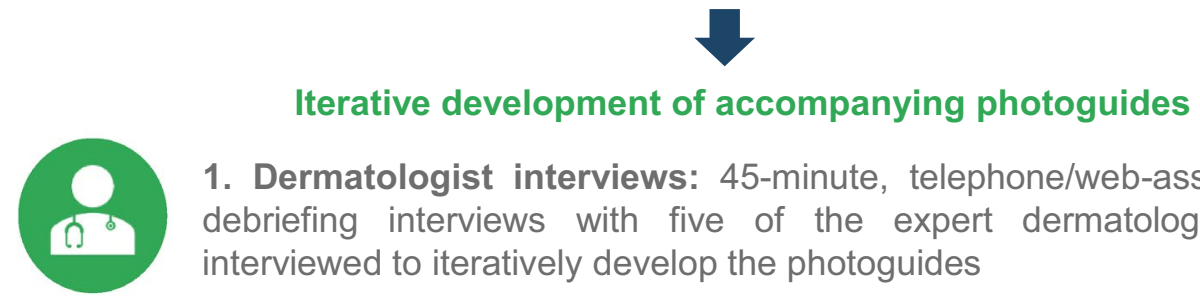

1. Dermatologist interviews: 45-minute, telephone/web-assisted cognitive debriefing interviews with five of the expert dermatologists previously interviewed to iteratively develop the photoguides

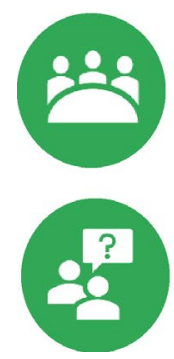

2. SPM: Lead AA expert (BAK) reviewed the final modifications made to the photoguides

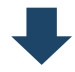

3. Patient Interviews: 45-minute, telephone/web-assisted cognitive debriefing interviews with 10 patients (not previously interviewed for this study)

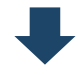

Final accompanying photoguides

Fig. 1 Iterative development of clinician-reported outcome (ClinRO) and patient-reported outcome (PRO) measures and accompanying photoguides. $A A$ alopecia areata

\subsubsection{Identification of Measurement Concepts}

Dermatologists described eyebrow and eyelash hair loss as facial defining features that changed patients' appearance and were difficult to conceal. In clinical practice, dermatologists assessed eyebrow hair loss primarily through consideration of coverage/continuity (i.e., absence of gaps), although some dermatologists considered density (fullness/thickness) and symmetry (across both eyes). Similarly, dermatologists assessed eyelash hair loss through consideration of continuity and symmetry.

Patients reported eyebrow hair loss $(n=28 / 35$ [80\%]) and/ or eyelash hair loss $(n=25 / 35$ [71\%]) among their three most bothersome signs/symptoms of AA. Patients described feeling embarrassed and self-conscious about their eyebrow hair loss and/or eyelash hair loss, expressing a desire to look "normal" or cosmetically acceptable. Some described concealing their eyebrow loss using makeup or hats/glasses. 
Patients who experienced eyebrow hair loss $(n=38)$ primarily described their eyebrow hair loss in terms of gaps/ patches, thinness, density and/or symmetry. Patients who experienced eyelash hair loss $(n=32)$ described the gaps as the most important aspect of their eyelashes to consider in evaluation.

Many $(n=22)$ patients also described functional impacts of eyebrow and/or eyelash hair loss, including problems with sweat $(n=6)$ or debris $(n=13)$ getting into their eyes, irritation from the wind $(n=2)$ or difficulty seeing because of glare or sunlight $(n=4)$. These patients described the sensation as "itching," "burning," "dry," or "like something being stuck in the eye."

All dermatologists described AA-related nail involvement as "pitting/pits" $(n=6)$, "breaking"/"splitting"/"brittleness" $(n=4)$, "dystrophy" $(n=2)$, "roughness" $(n=2)$, and "shedding" $(n=1)$. Patients with nail damage $(n=14)$ described their nails as pitted, split, bumpy, grainy, indented and/or feeling brittle, thin and/or weak. For some participants, nail damage was an aesthetic issue, whereas for others it caused functional problems (most did not experience any nail pain).
The SPM identified that the severity of nail damage was more important than the number of nails that were damaged.

From these insights, it was identified that PRO and ClinRO measures to assess eyebrow hair loss, eyelash hair loss, eye irritation and nail appearance should be developed as both dermatologists and patients considered these to be important signs/symptoms of AA. Patient and dermatologist quotes that support measurement concepts are presented in ESM 3.

\subsubsection{Development of ClinRO and PRO Measures to Assess Eyebrow and Eyelash Involvement}

The ClinRO measures for eyebrow hair loss and eyelash hair loss underwent three iterative revisions based on feedback provided in the dermatologist interviews (ESM 4 and 5). A four-option response scale was confirmed by dermatologists as appropriate to allow assessment of incremental severity on each ClinRO measure. The final version of each ClinRO measure was confirmed during the SPM and is presented in Table 1. These measures were also reviewed by patients,

Table 1 Final versions of ClinRO measures for eyebrow and eyelash hair loss, and PRO measures for eyebrows and eyelashes

\begin{tabular}{|c|c|}
\hline $\begin{array}{l}\text { ClinRO Measure for Eyebrow Hair Loss }{ }^{\mathbf{T M}} \\
\text { Examine both eyebrows from two feet away and } \\
\text { select the best response below. } \\
\square_{0} \text { The eyebrows have full coverage and no areas } \\
\text { of hair loss } \\
\square_{1} \text { There are minimal gaps in eyebrow hair and } \\
\text { distribution is even } \\
\square_{2} \text { There are significant gaps in eyebrow hair or } \\
\text { distribution is not even } \\
\square_{3} \text { No notable eyebrow hair }\end{array}$ & $\begin{array}{l}\text { PRO Measure for Eyebrows } \\
\text { Look at the hair in both of your eyebrows. Please } \\
\text { rate your eyebrows, as they look today. } \\
\text { This question asks about gap(s) in your } \\
\text { eyebrows or thinning in your eyebrows. } \\
\text { If you have gap(s) in your eyebrows and } \\
\text { thinning in your eyebrows, please choose your } \\
\text { answer based on the type of hair loss that } \\
\text { bothers you the most. } \\
\text { Please select one answer. } \\
\square_{0} \text { I have full eyebrows on each eye } \\
\square_{1} \text { I have a minimal gap(s) or a minimal amount of } \\
\text { thinning in at least one of my eyebrows } \\
\square_{2} \text { I have a large gap(s) or a large amount of } \\
\text { thinning in at least one of my eyebrows } \\
\square_{3} \text { I have no or barely any eyebrow hairs }\end{array}$ \\
\hline $\begin{array}{l}\text { ClinRO Measure for Eyelash Hair Loss } \\
\text { Examine the upper and lower eyelashes of both } \\
\text { eyes and select the best response option below. } \\
\square_{0} \text { The eyelashes form a continuous line along the } \\
\text { eyelids on both eyes } \\
\square_{1} \text { There are minimal gaps and the eyelashes are } \\
\text { evenly spaced along the eyelids on both eyes } \\
\square_{2} \text { There are significant gaps along the eyelids or } \\
\text { the eyelashes are not evenly spaced along the } \\
\text { eyelids } \\
\square_{3} \text { No notable eyelashes }\end{array}$ & $\begin{array}{l}\text { PRO Measure for Eyelashes } \\
\text { Look at your upper and lower eyelashes on both } \\
\text { your eyes. Please rate your eyelashes, as they look } \\
\text { today. } \\
\text { Please select one answer. } \\
\square_{0} \text { I have full eyelashes on each eyelid } \\
\square_{1} \text { I have a minimal gap or minimal gaps along the } \\
\text { eyelids } \\
\square_{2} \text { I have a large gap or large gaps along the } \\
\text { eyelids } \\
\square_{3} \text { I have no or barely any eyelash hair }\end{array}$ \\
\hline
\end{tabular}

The ClinRO Measure for Eyebrow Hair Loss ${ }^{\mathrm{TM}}$, ClinRO Measure of Eyelash Hair Loss ${ }^{\mathrm{TM}}$, PRO Measure for Eyebrows ${ }^{\mathrm{TM}}$, PRO Measure for Eyelashes $^{\mathrm{TM}}$ and accompanying photoguides (ESM 10) are subject to copyright owned by Eli Lilly and Company. Permission to use is granted under Creative Commons attribution-No derivatives 4.0 International License. Contact Eli Lilly and Company in the event of any proposed modification

$C l i n R O$ clinician-reported outcome, $P R O$ patient-reported outcome 
time permitting. In round 2, almost all patients who were consulted considered the final ClinRO Measure for Eyebrow Hair Loss ${ }^{\mathrm{TM}}(n=9 / 11)$ and the final ClinRO Measure for Eyelash Hair Loss ${ }^{\mathrm{TM}}(n=9 / 11)$ to be important for evaluating treatment outcomes.

The PRO measures were reviewed in dermatologist interviews and in the SPM; several changes were suggested to more closely align PRO measure wording with that of the ClinRO measures. Versions of the PRO measures were then tested with patients over two rounds of interviews with interim revision between rounds. The changes resulting from patients' feedback are reported in ESM 4 and 5. In round 2 interviews, all patients could provide responses to each PRO measure and generally understood the item wording and severity gradations within the response scale options. Therefore, no further changes or testing were required. The final version of each PRO measure is presented in Table 1.

\subsubsection{Development of a PRO Measure to Assess Eye Irritation}

A PRO measure to assess eye irritation was developed; a ClinRO measure was not developed because only a patient can report on the sensation of eye irritation. Dermatologist and patient feedback on the PRO measure to assess eye irritation is summarized in ESM 6. The final version of the PRO measure (Table 2) was confirmed by patients following round 2 interviews; all 15 patients could understand and interpret the item consistently with its intended meaning, and a few commented that the example descriptors provided in parentheses (itching, stinging, burning, dry) had helped them interpret the concept of eye irritation.

\subsubsection{Development of a ClinRO and PRO Measure to Assess Nail Involvement}

Dermatologist and patient feedback on the ClinRO and PRO measures to assess nail involvement is summarized in ESM 7. The final version of the PRO measure (Table 3 ) was confirmed by patients following round 2 interviews; all patients could understand and interpret the item consistently with its intended meaning, and the response options were generally understood and used appropriately. Patients consulted ( $n=10 / 11)$ also confirmed the relevance of the PRO and ClinRO measure, reporting that it was important for clinicians and patients to rate nail appearance.

\subsection{Development of Accompanying Photoguides}

\subsubsection{Sample}

Five of the expert dermatologists who participated in the COA development interviews (AA experience range 8-30 years [mean 17.7]) participated in interviews to develop and confirm the photoguides. Ten patients with AA with current eyebrow hair loss $(n=9[90 \%])$, eyelash hair loss $(n=8[80 \%])$ and/or nail damage $(n=3[30 \%])$, aged 21-54 years (mean 37.0) were also interviewed (see ESM 8).

\subsubsection{Drafting, Review and Finalization of Accompanying Photoguides}

The photoguides were developed to help raters derive their score, standardize ratings across raters and provide clear visual instructions for assessment of the observable signs of AA (eyebrow hair loss, eyelash hair loss and nail damage). A photoguide to accompany the PRO Measure for Eye Irritation ${ }^{\mathrm{TM}}$ symptom measure was not developed.

High-resolution photographs of patients' eyebrows, eyelashes and nails were obtained, with patient consent, from a specialist dermatology clinic. The eyebrow, eyelash and/or nail damage of these patients were rated by their dermatologists using the relevant ClinRO measure. Selected photographs were then edited using Adobe ${ }^{\circledR}$ Photoshop ${ }^{\circledR}$ software to depict greater or lesser severity (i.e., more or less hair loss/nail damage) corresponding to each measures' response options. The edited images were reviewed by dermatologists and iteratively modified throughout the dermatologist interview process to incorporate their feedback. Images were then approved by the lead AA expert and included in the photoguides, resulting in a final set of four severity images depicting three different patients/presentations for eyebrow

Table 2 Final version of the PRO Measure for Eye Irritation ${ }^{\mathrm{TM}}$

Please rate how irritated (e.g. itching, stinging, burning, or dry) either of your eyes have been in the past 7 days.

Please select one answer.

$\square$ My eyes have not been irritated

$\square$ My eyes have been a little irritated

$\square_{2}$ My eyes have been moderately irritated

$\square$ My eyes have been severely irritated

The PRO Measure for Eye Irritation ${ }^{\mathrm{TM}}$ is subject to copyright owned by Eli Lilly and Company. Permission to use is granted under Creative Commons attribution-No derivatives 4.0 International License. Contact Eli Lilly and Company in the event of any proposed modification

$P R O$ patient-reported outcome 
Table 3 Final versions of the ClinRO and PRO Measures for Nail Appearance

\section{ClinRO Measure for Nail Appearance ${ }^{\mathrm{TM}}$}

Examine the fingernails and toenails and select the best response below.

$\square_{0}$ Nails are not at all damaged (e.g. pitted, rough, brittle, split)

$\square$ At least one nail is a little damaged (e.g. pitted, rough, brittle, split)

$\square_{2}$ At least one nail is moderately damaged (e.g. pitted, rough, brittle, split)

$\square_{3}$ At least one nail is very damaged (e.g. pitted, rough, brittle, split) or subject has lost at least one nail
PRO Measure for Nail Appearance ${ }^{\mathrm{TM}}$

Examine your fingernails and toenails. Please rate your fingernails and toenails, as they look today.

Please select one answer.

$\square$ Nails are not at all damaged (e.g. pitted, rough, brittle, split)

$\square_{1}$ At least one nail is a little damaged (e.g. pitted, rough, brittle, split)

$\square$ At least one nail is moderately damaged (e.g. pitted, rough, brittle, split)

$\square$ At least one nail is very damaged (e.g. pitted, rough, brittle, split) or you have lost at least one nail

The ClinRO Measure for Nail Appearance ${ }^{\mathrm{TM}}$ and PRO Measure for Nail Appearance ${ }^{\mathrm{TM}}$ and accompanying photoguides (ESM 10) are subject to copyright owned by Eli Lilly and Company. Permission to use is granted under Creative Commons attribution-No derivatives 4.0 International License. Contact Eli Lilly and Company in the event of any proposed modification

ClinRO clinician-reported outcome, $P R O$ patient-reported outcome

loss and two different patients/presentations for eyelash hair loss and nail damage (photographs for eyelash hair loss and nail damage required enlargement for raters to be able to clearly view the detail presented). See ESM 9 for an illustrative figure of the development methodology and ESM 10 for the final photoguides.

The final images received a 98\% approval rating from the dermatologists and patients who reviewed them, confirming that the images appropriately depicted the severity levels they represented and that the photoguides provided a clear visual instruction for assessment. Nearly all dermatologists and patients felt that the photoguides helped them to derive ratings and would help to standardize ratings.

\section{Discussion}

This qualitative study confirmed that dermatologists and patients with AA consider eyebrow and eyelash hair loss as bothersome, necessitating treatment evaluation. Patients and dermatologists in this study also highlighted eye irritation as a physical impact of eyebrow/eyelash loss, endorsing eye irritation as an additional relevant concept for assessment. Both clinicians and patients also endorsed the assessment of nail appearance.

Investigator global assessment and PRO measures with accompanying photoguides have been used to assess eyebrows and eyelashes in clinical trials for other conditions [21, 22]; however, to our knowledge, these measures have not been designed to assess patients with AA. Thus, there was a need to develop appropriate measures for use in AA clinical trials.

The ClinRO and PRO measures were developed in line with FDA PRO Guidance for Industry [13]. Multiple rounds of concept elicitation and cognitive debriefing using industry standard methodology [14] were conducted with dermatologists and patients to craft measures that were informed by the intended population of use. This extensive testing resulted in measures of eyebrow hair loss, eyelash hair loss, nail appearance and eye irritation that are content valid (i.e., interpretable, relevant and usable) for their context of use and population of interest (i.e., clinical trials including patients with severe AA). However, the validation of new ClinRO and PRO measures requires a compilation of evidence [13]. Next, quantitative research will be conducted to psychometrically test and establish other aspects of validity and reliability and the ability of the measures to detect change.

Eyebrow hair loss, eyelash hair loss and nail appearance manifest differently from patient to patient, so describing different levels of severity within each ClinRO and PRO measure was a considerable challenge. To meet this challenge, and to support dermatologists and patients in providing their ratings, accompanying eyebrow, eyelash and nail photoguides were iteratively developed, incorporating feedback from expert dermatologists, and confirmed with patients. The interviewed dermatologists and patients deemed these photoguides useful in deriving their score, helping standardize ratings across raters and providing clear visual instruction for assessment.

Some limitations to the present study exist. Participants were recruited in North America and, thus, the findings may not be generalizable to other cultures and countries without further confirmatory work. Photographs for the photoguides were limited to those available with patient permissions, and the photos are of Caucasian models only.

\section{Conclusion}

The ClinRO Measure for Eyebrow Hair Loss ${ }^{\mathrm{TM}}$, ClinRO Measure of Eyelash Hair Loss ${ }^{\mathrm{TM}}$, ClinRO Measure for Nail Appearance $^{\mathrm{TM}}$, PRO Measure for Eyebrows ${ }^{\mathrm{TM}}$, PRO Measure for Eyelashes ${ }^{\mathrm{TM}}$, PRO Measure for Nail Appearance ${ }^{\mathrm{TM}}$ 
and PRO Measure for Eye Irritation ${ }^{\mathrm{TM}}$ and accompanying photoguides were developed to provide content-valid, clinically meaningful measures that reflect patients' and dermatologists' perspectives of AA. In conjunction with the Alopecia Areata Investigator Global Assessment ${ }^{\mathrm{TM}}$ (AA$\mathrm{IGA}^{\mathrm{TM}}$ ) [11] and Scalp Hair Assessment $\mathrm{PRO}^{\mathrm{TM}}$ [12] for scalp hair loss, these measures provide a comprehensive suite of assessments for key AA signs/symptoms.

Acknowledgements The authors thank the interviewed patients who clearly and courageously described their AA signs/symptoms and impacts and thoughtfully reviewed the measures/photoguides, the patients who bravely agreed to be photographed for the photoguides and the interviewed dermatologists for their insightful contributions to this study. The authors also thank the site coordinators and staff at the Department of Dermatology, University of California Irvine, Irvine, CA, USA; Department of Dermatology, Yale University School of Medicine, New Haven, CT, USA; Northwest Dermatology Institute, Portland, OR, USA; Dawes Fretzin Clinical Research Group, Indianapolis, IN, USA; and SKiN Centre for Dermatology, Peterborough, ON, Canada who facilitated the recruitment of and access to interviewed patients.

Author contributions KWW, HK, SK, NVJA, and JM contributed to the study conception and design, material preparation and data collection and analysis. BAK contributed to the study design and data collection and interpretation. NM contributed to data collection. JMK, FPN and YD contributed to data interpretation. The first draft of the manuscript was written by JM and HK, and all authors commented on previous versions of the manuscript. All authors read and approved the final manuscript.

Copyright The ClinRO Measure for Eyebrow Hair Loss ${ }^{\mathrm{TM}}$, ClinRO Measure of Eyelash Hair Loss ${ }^{\mathrm{TM}}$, ClinRO Measure for Nail Appearance $^{\mathrm{TM}}$, PRO Measure for Eyebrows ${ }^{\mathrm{TM}}$, PRO Measure for Eyelashes ${ }^{\mathrm{TM}}$, PRO Measure for Nail Appearance ${ }^{\mathrm{TM}}$ and PRO Measure for Eye Irritation $^{\mathrm{TM}}$ measures and accompanying photoguides are subject to copyright owned by Eli Lilly and Company. Permission to use is granted under Creative Commons attribution-No derivatives 4.0 International License. Contact Eli Lilly and Company in the event of any proposed modification.

Data availability The datasets generated during and/or analyzed during the current study are not publicly available because of the confidential nature of the information. However, the corresponding author can be contacted for any data-related questions.

\section{Declarations}

Funding This study was funded by Eli Lilly and Company.

Conflict of interest Helen Kitchen, Sarah Knight, Natalie V.J. Aldhouse, and Jake Macey are employees of DRG Abacus, a health economic and outcomes research consultancy that consults with various pharmaceutical companies. Kathleen W. Wyrwich was an employee and stockholder at Eli Lilly and Company when this research was conducted; she is now an employee and stockholder at Pfizer Inc. Fabio P. Nunes, and Yves Dutronc are employees and stockholders at Eli Lilly and Company. Dr. Brett King has served on advisory boards and is a consultant and clinical trial investigator for Eli Lilly and Company; he has served on advisory boards and/or is a consultant and/or is a clinical trial investigator for Aclaris Therapeutics Inc, Arena Pharmaceuti- cals, Bristol-Meyers Squibb, Concert Pharmaceuticals Inc, Dermavant Sciences Inc and Pfizer Inc. Natasha Mesinkovska has no potential conflicts of interest. Eli Lilly and Company have commissioned DRG Abacus, Dr. Justin M. Ko and Dr. Brett A. King to consult on clinical outcomes assessment strategies for AA.

Research involving human participants The interview guides and methodology for this study were approved by the Human Research Ethics Committee Western Institutional Review Board (ref \#20171820).

Informed consent Informed consent was obtained from all individual participants included in the study. The photographs used in the photoguide were obtained separately from this study and its ethics committee approval; however, consent and release was obtained from patients to disclose their photographs.

Open Access This article is licensed under a Creative Commons Attribution-NonCommercial 4.0 International License, which permits any non-commercial use, sharing, adaptation, distribution and reproduction in any medium or format, as long as you give appropriate credit to the original author(s) and the source, provide a link to the Creative Commons licence, and indicate if changes were made. The images or other third party material in this article are included in the article's Creative Commons licence, unless indicated otherwise in a credit line to the material. If material is not included in the article's Creative Commons licence and your intended use is not permitted by statutory regulation or exceeds the permitted use, you will need to obtain permission directly from the copyright holder. To view a copy of this licence, visit http://creativecommons.org/licenses/by-nc/4.0/.

\section{References}

1. Food and Drug Administration (FDA). Patient-focused drug development: collecting comprehensive and representative input guidance for industry, Food and Drug Administration staff, and other stakeholders. 2018. https://www.fda.gov/downloads/drugs/guida ncecomplianceregulatoryinformation/guidances/ucm610442.pdf. Accessed Oct 2018.

2. Olsen E, Hordinsky M, McDonald-Hull S, Price V, Roberts J, Shapiro J, et al. Alopecia areata investigational assessment guidelines. J Am Acad Dermatol. 1999;40(2):242-6.

3. Olsen EA, Hordinsky MK, Price VH, Roberts JL, Shapiro J, Canfield $\mathrm{D}$, et al. Alopecia areata investigational assessment guidelines—part II. J Am Acad Dermatol. 2004;51(3):440-7.

4. Solomon JA. Development of uniform protocol for alopecia areata clinical trials. J Investig Dermatol Symp Proc. 2015;17(2):63-6.

5. Finner AM. Alopecia areata: clinical presentation, diagnosis, and unusual cases. Dermatol Ther. 2011;24(3):348-54.

6. Anderson I. Alopecia areata: a clinical study. BMJ. 1950;2(4691):1250-2.

7. Wyrwich KW, Kitchen H, Knight S, Aldhouse NVJ, Macey J, Nunes F, et al. A qualitative interview study to understand the psychosocial burden of alopecia areata. In: 28th congress of European academy of dermatology and venereology, Madrid, Spain; 9-13 Oct 2019.

8. Hunt N, McHale S. The psychological impact of alopecia. BMJ. 2005;331(7522):951-3.

9. Sadr J, Jarudi I, Sinha P. The role of eyebrows in face recognition. Perception. 2003;32(3):285-93.

10. Chelidze K, Lipner SR. Nail changes in alopecia areata: an update and review. Int J Dermatol. 2018;57(7):776-83.

11. Wyrwich KW, Kitchen H, Knight S, Aldhouse NVJ, Macey J, Nunes FP, et al. The Alopecia Areata InvestigatorGlobal 
Assessment (AA-IGA ${ }^{\mathrm{TM}}$ ) scale: a measure for evaluating clinically meaningful success in clinical trials. Br J Dermatol. 2020. https://doi.org/10.1111/bjd.18883.

12. Wyrwich K, Kitchen H, Knight S, Aldhouse N, Macey J, Nunes F, et al. Development of the scalp hair assessment Patient-Reported Outcome $^{\mathrm{TM}}$ (PRO) measure for alopecia areata. Br J Dermatol. 2020. https://doi.org/10.1111/bjd.19024.

13. Food and Drug Administration (FDA). Guidance for industry: Patient-reported outcome measures: Use in medical product development to support labeling claims. 2009. https://www.fda.gov/ media/77832/download. Accessed June 2019.

14. Patrick DL, Burke LB, Gwaltney CJ, Leidy NK, Martin ML, Molsen E, et al. Content validity—establishing and reporting the evidence in newly developed patient-reported outcomes (PRO) instruments for medical product evaluation: ISPOR PRO Good Research Practices Task Force report: part 2-assessing respondent understanding. Value Health. 2011;14(8):978-88.

15. Willis GB. Cognitive interviewing: a tool for improving questionnaire design. Thousand Oaks: Sage Publications; 2004.

16. Braun V, Clarke V. Using thematic analysis in psychology. Qual Res Psychol. 2006;3(2):77-101.
17. Guest G, MacQueen KM, Namey EE. Applied thematic analysis. Thousand Oaks: Sage Publications; 2011.

18. Fusch PI, Ness LR. Are we there yet? Data saturation in qualitative research. Qual Rep. 2015;20(9):1408-16.

19. Turner-Bowker DM, Lamoureux RE, Stokes J, Litcher-Kelly L, Galipeau N, Yaworsky A, et al. Informing a priori sample size estimation in qualitative concept elicitation interview studies for clinical outcome assessment (COA) instrument development. Value Health. 2018;21(7):839-42.

20. Ritchie J, Lewis J, Nicholls CM, Ormston R. Qualitative research practice: a guide for social science students and researchers. Thousand Oaks: Sage Publications; 2013.

21. Ahluwalia GS. Safety and efficacy of bimatoprost solution $0.03 \%$ topical application in patients with chemotherapy-induced eyelash loss. J Investig Dermatol Symp Proc. 2013;16(1):S73-S7676.

22. Carruthers J, Beer K, Carruthers A, Coleman WP III, Draelos ZD, Jones D, et al. Bimatoprost $0.03 \%$ for the treatment of eyebrow hypotrichosis. Dermatol Surg. 2016;42(5):608-17.

\section{Affiliations}

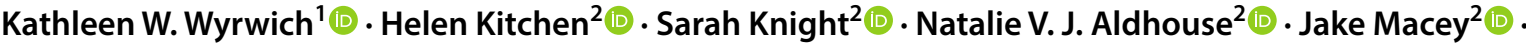

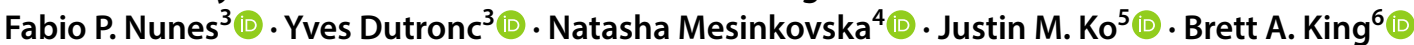

\author{
Kathleen W. Wyrwich \\ kathy.wyrwich@gmail.com \\ Helen Kitchen \\ hKitchen@teamdrg.com \\ Sarah Knight \\ sknight@teamdrg.com \\ Natalie V. J. Aldhouse \\ naldhouse@teamdrg.com \\ Jake Macey \\ jmacey@teamdrg.com \\ Fabio P. Nunes \\ nunes_fabio_p@lilly.com \\ Yves Dutronc \\ dutronc_yves@1illy.com \\ Natasha Mesinkovska \\ nmesinko@hs.uci.edu \\ Justin M. Ko \\ jmko@stanford.edu
}

1 Patient-Focused Outcomes Center of Expertise, Eli Lilly and Company, Lilly Corporate Center, Indianapolis, IN 46285, USA

2 Clinical Outcomes Assessment, DRG Abacus, The Lexicon, Mount Street, Manchester M2 5NT, UK

3 Lilly Bio-Medicines, Eli Lilly and Company, Eli Lilly and Company, Lilly Corporate Center, Indianapolis, IN 46285, USA

4 University of California Irvine Dermatology Clinical Research Center, Hewitt Hall Building, 843 Health Sciences Road, Room 1001, Irvine, CA 92697, USA

5 Stanford Dermatology, Stanford University School of Medicine, 291 Campus Drive, Li Ka Shing Building, Stanford, CA 94305-5101, USA

6 Department of Dermatology, Yale School of Medicine, 333 Cedar Street, LMP 5040, New Haven, CT 06520, USA 